\title{
Algunas variantes textuales propuestas por Juan de Fonseca en su comentario a Andria
}

\author{
Milagros DEL Amo LoZANO \\ Universidad de Murcia \\ latinmila@hotmail.com \\ $M^{\mathrm{a}}$. Teresa BELTRÁN NOGUER \\ Universidad de Murcia \\ materesa@um.es
}

Recibido: 14 de febrero de 2011

Aceptado: 9 de marzo de 2011

\section{RESUMEN}

En este artículo se analizan algunas cuestiones textuales que Juan de Fonseca y Figueroa trata en su comentario a la Andria terenciana. Su preocupación por el texto atañe no solo a la obra que es objeto de su estudio, sino que también aprovecha para hacer crítica y sugerencias a propósito de algunos textos que aduce en la explicación del poeta. Se analizan las fuentes de esas lecturas, los argumentos en los que el humanista se apoya para defenderlas y la pervivencia que luego han tenido.

Palabras clave: Terencio. Fonseca. Andria. Crítica textual.

del Amo Lozano, M. - Beltrán Noguer, Ma T., «Algunas variantes textuales propuestas por Juan de Fonseca en su comentario a Andria», Cuad. Fil. Clás. Estud. Lat. 31.1 (2011) 147-165.

\section{Some textual variants proposed by Juan de Fonseca in his commentary to Andria}

\begin{abstract}
This article analyses some textual questions which Juan de Fonseca y Figueroa deals with in his commentary on Terence's Andria. He concerns himself not only with the work he is studying but also takes the opportunity to criticize and make suggestions regarding some of the texts he uses in his explication of the poet. The sources of these readings, the argument used by the humanist to defend them and their subsequent trajectory are analyzed.
\end{abstract}

Keywords: Terencio. Fonseca. Andria. Textual criticism.

Del Amo Lozano, M. - Beltrán Noguer, Ma T., «Some textual variants proposed by Juan de Fonseca

in his commentary to Andria», Cuad. Fil. Clás. Estud. Lat. 31.1 (2011) 147-165. 
Don Juan de Fonseca y Figueroa (Badajoz 1585-Madrid 1627), como es sabido, fue una persona de gran prestigio e influencia en su época; pertenecía a una familia noble: su hermano, por ejemplo, fue marqués de Orellana; alcanzó cargos relevantes; fue sumiller de Cortina del rey Felipe IV y embajador en Parma; entre sus amigos destacaron escritores, pintores, o humanistas; valgan de ejemplo, Velázquez -del que fue de alguna manera mecenas-, Lópe de Vega, Octavio Corsini, Pacheco, Vera y Zúñiga, Francisco de Calatayud, Francisco de Rioja, González de Salas o Gaspar Scioppius, por citar solo unos nombres; persona de gran cultura, también gozó de bastante autoridad intelectual; consta, en efecto, que le hacían consultas personas muy eruditas.

Él mismo era ciertamente un erudito, al que pocas cosas le eran ajenas, pues le interesaban desde los textos clásicos hasta la poesía castellana, y de modo especial los poetas andaluces, y en particular los sevillanos, sin pasar por alto su interés por las inscripciones latinas o por la pintura; fue, podríamos decir, un «humanista», al que no le alcanzó la suerte de que su obra escrita llegara a la imprenta; razones distintas y complementarias pudo haber para que esto ocurriera, una pudo ser que no juzgó dispuestas para la imprenta ninguna de ellas, o bien que las muchas ocupaciones y responsabilidades le impidieron disponer del tiempo requerido para poner el punto final a las mismas.

Cuáles eran esas obras lo sabíamos por Nicolás Antonio (1783, I, p.691), quien también ofrece los datos fundamentales sobre su vida, aunque algunos de ellos se han ido concretando y ampliando después.

Tiene mucha utilidad, para acercarnos a la personalidad de este humanista, el juicio que hace de él, transmitiendo las palabras de Gonzalo de Correa, cuando afirma que era inter literatos literatissimus, inter notabiles notabilissimus, inter utrosque praestantissimus $^{1}$. Esta consideración hacia él de Correa explica que le dedique dos obras $^{2}$.

Un hombre, por tanto, bien valorado en su tiempo, al que también Tamayo de Vargas o Ramírez de Prado le dedicaron elogiosos apelativos ${ }^{3}$.

Respecto a los datos que ofrecía Nicolás Antonio se ha ido avanzando mucho en la biografía de don Juan de Fonseca; y ello gracias a lo que ofrecen algunos manus$\operatorname{critos}^{4}$, y a noticias que hallamos en cierta bibliografía que, aun no siendo específica

${ }^{1}$ Cf. N. Antonio (1783), loc. cit.

2 En el ms. 161 de la Biblioteca Nacional están encuadernadas las dos obras que le dedica (una impresa y otra manuscrita); una de ellas es la Commentatio seu Declaratio ad illud Geneseos (Correa 1622); cuya dedicatoria reza: Domno Ioanni Fonsekio Figheroo Hispalitanae Ecclesiae Canonico et Scholastico amplissimo Regi Filippo iiii. A Cortinas, a Magistro Gonzalo Corrêas observantiae testimonium. La otra obra que le dedica es una especie de carta, de título Nominis Dei vera ethymologia et significatio, ad Ioannem Fonsekium Figheroum, a Magist. Gonzalo Corrêas linguarum Graecae et Hebraicae Salmanticae primario investigata et exposita, que aparece citada, v. gr., en Ajo y Sáinz de Zúñiga (1972, 8, p.268), donde se afirma que data de 1622.

${ }^{3}$ Nobile iuventutis Hispaniae decus dice de él Ramírez de Prado, cf. La Barrera (1867, p.317).

${ }^{4}$ Por ejemplo, en el manuscrito 5781 de la Biblioteca Nacional (antes Q-87) se hallan escritos suyos, y de él se puede extraer amplia información sobre Fonseca y sus amigos; hay muchas cartas de algunos de ellos dirigidas a Fonseca; de G. Scioppius, v.gr., se halla una en el fol. 103v. 
sobre él, transmite datos importantes ${ }^{5}$, así como en los libros de matrículas de Salamanca ${ }^{6}$ o de Sevilla ${ }^{7}$, o lo que extraemos de la correspondencia con sus amigos ${ }^{8}$ y de su propia obra. Podemos decir, por ejemplo, que nació en Badajoz y no en Sevilla, según consta en expediente de limpieza de sangre ${ }^{10}$, el cual también aporta otros datos de su biografía ${ }^{11}$.

En definitiva, estamos ante un personaje del siglo XVII que escribió sobre diferentes autores, que participó en las discusiones humanistas - muchas, como veremos, de asuntos «textuales», que también hizo incursiones en la historia de España, en las inscripciones ${ }^{12}$, en la poesía, y en la pintura.

En cuanto a las obras, Nicolás Antonio ofrecía -hemos dicho- una relación que hoy se sabe incompleta, él ya menciona entre otros sus trabajos sobre Claudiano ${ }^{13}$, Terencio o Séneca ${ }^{14}$, una obra sobre pintura ${ }^{15}$, una defensa del Marcial de Ramírez de Prado ${ }^{16}$ y otra que realizó para defender algunas correcciones textua-

${ }^{5}$ Es el caso de obras sobre hombres de su tiempo, sobre Francisco de Rioja u otras sobre Velázquez, personajes que tuvieron relación con Fonseca; destacan los datos que hallamos en La Barrera (1867); $c f$. también López (1984).

${ }^{6}$ Sabemos por los Libros de Actas de dicha Universidad que estudió allí del 1604 al 1608, aunque también sabemos que en ese tiempo hizo varios viajes, a Madrid especialmente, $c f$. AUSA 314, 315,316 y 317. En esta Universidad coincide con Baltasar de Céspedes, Roque de Vergas, Solórzano y Ramírez de Prado, entre otros; en ella pronunció en 1609 (anno domini MDCVIII) su Oratio recitata pro obtinenda bacchalauri licencia in Salmanticensi academia, que leemos en el manuscrito de la Biblioteca Colombina; $c f$. infra.

${ }^{7}$ En el Libro de Matrículas de la Universidad de Sevilla, en abril del 1607, hallamos: «don Juan de Fonseca, natural de Badajoz, canónigo de la Sta. Iglesia de Sevilla juro ocho del dicho y truxo carta de examen»; cf._AHUS libro 482, fol. 42r.

${ }^{8}$ Muchas de las cartas a personajes como Octavio Corsini, Francisco de Eraso, Francisco de Calatayud, José de Salas o G. Scioppius se hallan en el ms. 12639 de la Biblioteca Nacional, fols. 214-246.

${ }^{9}$ Se explicaría mejor, por ejemplo, su interés en datos biográficos de Pedro de Valencia: siendo este de Zafra, se trataba de un extremeño como él; si bien el hecho de que hubiera escrito una obra - Academica- dedicada a su tío materno podía ser suficiente para prestar atención a este humanista (cf. Moya 1988, p.12).

${ }^{10}$ Cf. López Navío (1964, pp.4 y 8-9). También en las Actas de matrículas de la Universidad de Salamanca arriba citadas se le menciona como natural de Badajoz.

11 Para más datos sobre su biografía, puede verse La Barrera (1867, pp.292-319), López Navío (1964), Amo-Moya (2008); y, sobre todo, Moya (1986).

12 Además de sus Observationes adversus Gruterum, él mismo participó en el proyecto de una inscripción latina -en colaboración con Francisco de Calatayud- para un monumento que el Rey ordenó que se construyese en el Sitio de Campillo, cercano al Escorial. La inscripción puede verse en el fol. 133 del ms. 5781, fechada el 17 de septiembre de 1623.

${ }^{13}$ Ad Claudiani de Raptu Proserpinae notulas inceptas. Sobre estas notas el profesor J. F. Ortega Castejón presentó una comunicación en el X Simposio de Estudios Clásicos, Tarragona, 1990.

${ }^{14}$ In Senecae Epistolam XVIII lib. II.

15 De veteri pictura.

${ }^{16}$ Fonseca escribió contra la crítica que Theodorus Marcilius, bajo el seudónimo de Claudius Mussambertus, había realizado a la obra sobre Marcial de Ramírez de Prado (Hypomnemata ad lib. Spectaculorum et quatuor primus Epigrammatom M.V. Martialis < .. > Parisiis, apud Michaelem Somnium, 1607), que tituló In L. Ramiresii ad M.V. Martialem hypomnemata (Paris, exc. Dionysius Langlois, 1607). Fue una defensa que el propio Ramírez agradeció. Estas son las palabras de Nicolás Antonio: Pro Laurentio Ramirez de Prado adversus Mussambertium, seu Theodorum Marsilium. Meminit hujus libri D. Laurentius ipse in Pentecontarcho, cap. XLIV, $c f$. N. Antonio, loc. cit. 
les de Lipsius ${ }^{17}$, además de sus escritos sobre Pseudo Dextri y Pseudo Maximi y unas observationes contra Gruterius ${ }^{18}$. También alude a la carta en que Juan Bautista Salazar le hace una consulta sobre un lugar de Catulo $66^{19}$. De ellas la más importante, sin duda, es el comentario, o Notas, como Fonseca las llama, que dedica a la terenciana Andria, de la que nos vamos a ocupar.

Algunas de estas obras, como su defensa de Ramírez de Prado contra los ataques de Mussambertus o la de las enmiendas de Lipsius fueron muy conocidas en su tiempo.

El descubrimiento de los manuscritos ${ }^{20}$ hace ver que la mayoría de dichas obras no se ocupan de todo el conjunto, sino que son unas relativamente breves anotaciones sobre algunos lugares; por ejemplo, el comentario a Claudiano atiende solo al prefacio, la De criticis disceptatiuncula, a algunas lectiones de las tragedias de Séneca.

Se ha ido ampliando la «lista» y el conocimiento de esas obras, y, además de completarse la relación de ellas, va viendo la luz una parte de su producción ${ }^{21}$.

Así se ha sabido que comentó a Garcilaso ${ }^{22}$ y la Jerusalén de Lope ${ }^{23}$, que es autor de seis dísticos que, dedicados a su amigo Ramírez de Prado, preceden al Pentekontarchos de este ${ }^{24}$. También hay constancia de que escribió otras que hoy no conservamos, al menos sobre Plinio y sobre Petronio ${ }^{25}$.

Las notas a la Andria de Terencio son, sin duda, su obra más importante, y una de las primeras que escribió ${ }^{26}$. El interés por las comedias de Terencio siempre fue grande; los comentarios de Donatus o Eugraphius lo avalan; ese interés no desapareció en épocas sucesivas y, por lo que respecta a España, pese a su mengua de popula-

17 Se trata de la De criticis Disputatiunculam inter Neotericum Scriptorem, que en realidad se llama De criticis Disceptativncvla inter Neotericum Scriptorem, et ...; sobre esta obra la profesora F. Moya del Baño presentó una comunicación en el XII Congreso de Estudios Clásicos, cuyo título era «Fonseca y Figueroa y el texto de las Tragedias de Séneca»».

18 Observationes nostras adversus Gutherium.

${ }^{19}$ Un análisis de la lectio que en esta carta se debate, puede verse en Ortega (1989) y Charlo (2007).

${ }^{20}$ Especialmente el ms 5781 citado en la nota 4, pero también otros como el 12639, el 388 y el 161.

${ }^{21}$ Respecto a otras obras del humanista, puede verse un «catálogo» en La Barrera (1867, pp.306-319), sobre todo, y, más reciente y actualizado, en Moya (1986, pp.208-211); afirma La Barrera que de todo lo que escribió solo fueron editados en la antigüedad dos pequeños fragmentos; gracias a un Proyecto de Investigación, cuyo Investigador Principal es la Dra. Francisca Moya del Baño, se van dando a la luz algunas de sus obras: $c f$. Moya (1986), Moya-Beltrán (1987-1989), Moya-Fortuny (1990) y Miralles (2010).

${ }^{22}$ Lo que Fonseca leyó y aclaró de Garcilaso ha sido estudiado por la prof. Moya en un ilustrador artículo que ofrece mucha información acerca de este canónigo hispalense; $c f$. Moya (1986).

${ }^{23}$ Dichas notas ya fueron publicadas, sin atribuírsele a él, por Entrambasaguas; más recientemente las profesoras F. Moya y $\mathrm{M}^{\mathrm{a}} \mathrm{T}$. Beltrán han revisado el trabajo del humanista recuperando su autoría; $c f$. MoyaBeltrán (1987-1989).

${ }^{24}$ El profesor J. C. Miralles ha estudiado esta faceta del polifacético Fonseca: $c f$. Miralles (2010).

${ }^{25}$ Queda reflejado en la obra de González de Salas sobre el Satyricon que se había ocupado de Petronio: cf. Moya-Fortuny (1990); y, con toda seguridad, según se desprende de otras obras suyas, y de cartas y testimonios de otros, aunque no nos haya llegado, trabajó sobre Plinio: $c f$. Moya (2006).

${ }^{26}$ Fonseca redacta estas notas en 1606, según se desprende de una carta-fechada el 2 de diciembre de ese año, que precede a este trabajo y que está dirigida a don J. de Solórzano y Pereira, a quien dedica la obra; la edición y traducción de esta carta, así como la respuesta del amigo, se halla en Amo-Moya (2008). 
ridad a finales del siglo XVI, vuelve a resurgir en el XVII entre los eruditos ${ }^{27}$, siendo un claro ejemplo el de Fonseca.

Las Notas se encuentran manuscritas en la Biblioteca Colombina ${ }^{28}$, y, como venimos afirmando, es la obra más amplia y más acabada ${ }^{29}$. Aborda en ella un comentario del texto terenciano ${ }^{30}$ en que se ocupa de muchos aspectos que el humanista considera dignos de ser analizados; no se trata de un comentario a la obra completa, sino de anotaciones a algunos lugares que él ha seleccionado por diversas razones ${ }^{31}$; aunque abarquen distintos campos del saber, atiende más bien al contenido, poniéndolo en parangón a menudo con el de las comedias que le sirvieron de fuente ${ }^{32}$, así como mostrando los paralelos de autores clásicos y modernos que pueden dar luz a esta obra de Terencio ${ }^{33}$, y tratando de «rellenar» las lagunas que observa.

Para llevar a cabo su obra contaba con unos precedentes, ya que un buen número de humanistas se habían ocupado de ilustrar al poeta y, como era habitual, las tendría en cuenta; él mismo confiesa, en una hoja que precede a esta obra, que se sirvió de una edición parisina de Stephanus que contenía los comentarios de Donatus y Eugraphius ${ }^{34}$

\footnotetext{
${ }^{27}$ Recordamos el muy clarificador trabajo del prof. L. Gil (1984). Allí se nos recuerda que fue editado, comentado y traducido por autores hispanos por primera vez en este siglo XVI; fue Pedro de Figueroa quien primero lo explicó (de 1559 son sus Enarrationes vere aureae in P. Terentii Andriam et Eunuchum. Valentiae, ex typographia Petri a Huete, MDLXIX); y Pedro Simón Abril quien en 1577 hizo pública su traducción de las seis comedias de Terencio (Las seys Comedias de Terencio, escritas en latín y traduzidas en vulgar Castellano...); cf. Gil (1984, especialmente pp.99 y ss). Así pues, tenía nuestro humanista antecedentes hispanos en quien fijar su mirada; en efecto, la edición de las Enarrationes del humanista valenciano -anuncia él mismole servirán de base, junto con otras ediciones, para sus Notae.

${ }^{28}$ Ms. Biblioteca Colombina 57-3-24 (antes ms. n 21, y 83, 3-19), fols. 163-221. Además en el manuscrito 5781 hallamos tres páginas (folios $156 \mathrm{r}, 157 \mathrm{r} \mathrm{y} \mathrm{v}$ ), con una pequeña anotación a Andria, con una letra que podría ser de otra mano (salvo que Fonseca la hubiera realizado de modo rápido o descuidado), lo cual muestra el interés por las aclaraciones de esta obra; se comentan allí algunos lugares de Andria; uno de los cuales, de tipo textual, coincide con el que hay en la obra más amplia que aquí estamos tratando.

${ }^{29}$ El manuscrito mismo manifiesta que la obra estaba terminada, y lo confirma el hecho de enviarla a su amigo J. de Solórzano para que la «supervise» y le corrija lo que estime conveniente. Posiblemente la falta de tiempo para incluir las observaciones que el amigo le hizo impidió el envío a la imprenta.

${ }^{30}$ Recordamos que este no es el único poeta sobre el que trabajó: hemos hablado de su obra sobre Claudiano, Garcilaso o Lope de Vega; igualmente de su interés por la poesía que le llevó a preparar una antología de poetas andaluces, e incluso quizá pensara abordar una antología más amplia, según puede deducirse de una parte de las notas a la Jerusalén de Lope (cf. Moya-Beltrán 1987-1989, p.999). Y también hemos señalado que él mismo escribió poesía.

${ }^{31}$ Manifiesta, v. gr., que hubiera supuesto un comentario demasiado largo corregir todos los textos griegos que aporta para ilustrar la comedia, o que consideraba importante atender a asuntos como los relacionados con las costumbres, especialmente en los lugares en que nada había dicho Donato; (Amo-Moya 2008, pp.306-307).

32 Esto es, Andria y Perinthia de Menandro.

${ }^{33}$ Entre las citas que aportan claridad a lo que en Terencio hay, ocupan un lugar importante las de la comedia y, sobre todo, las del propio poeta, (Amo-Fortuny 2005).

${ }^{34}$ Quizá la de 1529: P. Terentii Comoediae sex, tum ex Donati commentariis, tum ex optimorum, praesertim veterum, exemplarium collatione, diligentius quam vnquam antehac, emendatae. Aelii Donati antiquissimi et celeberrimi grammatici in easdem, quicunque extant, commentarii, ex veteri codice manu descripto, graecis etiam prepositis, accurate castigati. Calphurnii in tertiam comoediam doctissima interpretartio. Eorum, quae in commentariis sparsim annotata sunt, index amplissimus. Parisiis. Ex officina Roberti Stephani. MDXXIX.
} 
y una de Pedro de Figueroa ${ }^{35}$. Transmite a veces lo que otros comentaristas han dicho sobre esta comedia, ya sea para mostrarse de acuerdo con ellos ya para -la mayoría de las veces- criticarlos, pero -adelantamos- hay mucho en esta obra de Fonseca que no hemos encontrado en comentaristas anteriores ${ }^{36}$.

Ya hemos descrito en otro lugar cuál es la naturaleza de las notas de nuestro Fonseca y hemos dado cuenta, por ejemplo, de que son más extensas las que tienen que ver con explicaciones de realia; pero hay muchas muy breves en las que se ocupa del léxico, y otras que tienen que ver con la sintaxis, con la fonética o con la métrica (muy escasas estas), y también con la retórica ${ }^{37}$.

En esta ocasión nos vamos a centrar en la preocupación de Fonseca por el texto $^{38}$, tal como lo muestra en las Notae. Su afán por fijarlo, por que se use la grafía adecuada, se enmiende una lectura que él juzga errónea, o se mejore el verso con lo que otros o él mismo han sugerido, tiene una presencia considerable en este trabajo de Fonseca: le lleva no solo a mostrar interés en que la obra que comenta, Andria, quede con las lectiones más oportunas, sino que esa labor -podríamos decir- de 'crítico textual' le hace proponer correcciones a algunos lugares de los textos que cita para explicar lo que pretende, e incluso a algún otro que aparece incluido dentro de alguna cita de las que él proporciona. Podemos así ver que no se ha limitado a $A n-$ dria, ni siquiera a Terencio, sino que ha enmendado lugares de Diomedes, Lucilio, Marcial, Mela, Plauto, Séneca, Servio y Símaco; y que tampoco omite hacer correcciones a algunos textos griegos aportados ${ }^{39}$, así como a alguna glosa ${ }^{40}$.

\footnotetext{
${ }^{35} \mathrm{El}$ profesor J.D. Castro de Castro ha estudiado en profundidad la obra de este humanista valenciano, y en su trabajo presta atención a las ediciones y comentarios humanísticos anteriores; $c f$. Castro (2007).

${ }^{36}$ Además de los comentaristas antiguos, Donatus y Eugraphius, se habían ocupado de Andria con anterioridad, ya sea para hacer solo pequeñas anotaciones o comentarios más amplios, entre otros, Ascensius, Antesignanus, Doletus, Erasmus, Faernus, Goveanus, Guido Juvenalis Cenomanus, Lindenborgius, Petrus Marsus, Menenius, Muretus o Pedro de Figueroa. Una lista detallada de todos los autores que hasta ese momento habían dicho algo sobre Terencio se halla en la edición de Mureto de 1580, pp.124-125. En el trabajo del prof. Castro sobre las Enarrationes de Pedro de Figueroa se halla un buen análisis de las aclaraciones de Terencio que precedieron a la obra de este humanista; $c f$. Castro (2007).

${ }^{37}$ Sobre este comentario en general y sobre algunos aspectos en particular, $c f$. Beltrán (2007-2008), Amo-Fortuny (2005), Amo-Fortuny (2006), Amo-Moya (2008) y Amo (2009).

${ }^{38}$ Una preocupación que, por otra parte, nada extraña en los humanistas, ya que todos se muestran interesados en la edición de los textos. En el caso de Fonseca esta preocupación se halla presente -lo hemos referido- en la correspondencia que mantiene con otros humanistas: además de la ya aludida discusión de Catulo 66, sabemos que prestó ayuda a González de Salas sobre algunas lectiones de Petronio, que corrigió con «inflexible severidad» unos versos de Vera y Zúñiga ( $c f$. La Barrera, 1987), que algunas lecturas difíciles de la Naturalis historia le hicieron acercarse muchas veces al texto de Plinio -en dos cartas a Francisco de Calatayud hace sendas correcciones, a nat. 29.72 (parcitur y no pascitur) y a 8-194, (Sancus y no Sanctus o San$g u s$ ) - y probablemente las dificultades que allí encontró le indujeron a no publicar su trabajo (dato que conocemos por cartas de G. Scioppius o de Francisco de Rioja, ambas de 1616, u otras muchas dirigidas a Martín Velasco). Así mismo alguna de sus obras completa tiene como objeto fundamental este quehacer filológico (cf. su pro Laurentio Ramirez de Prado o su Disceptatiuncula inter Neotericum Scriptorem, citadas en las notas 16 y 17$)$.

${ }^{39}$ En las notas a los versos 161, 473, 483 y 726 vemos las correcciones a los textos de Esquines, Píndaro, Menandro, Libanio y Pólux, respectivamente.

${ }^{40}$ Notas a los vv. 79 y 442.
} 
Pero en la mayoría de los casos atiende al texto de Terencio; o mejor, todas las alusiones a diversas lectiones de Terencio se refieren a Andria, el poema objeto de su comentario, excepción hecha de una corrección (restitui dice), que, en la nota al verso 75 de la misma Andria, hace a Heautontimorumenos 282-285, unos versos que Fonseca considera corruptos ${ }^{41}$.

Vamos a ofrecer una descripción general de qué lugares propone enmendar, o acerca de cuáles ofrece varias lecturas, y luego nos detendremos en algunas de esas Notae $^{42}$.

Sobre Andria trata cuestiones textuales que son de muy distinto calado; las hay de tipo meramente gramatical (son variantes que tienen que ver con la fonética, la morfología o la sintaxis), así en $143^{43}$ en que sugiere que el dativo illi, de desinencia pronominal, sea sustituido por illo ${ }^{44}$, o en 437 en que ve mejor pote que potis, argumentando las posibilidades de la diferencia entre el género de uno y otro con varias citas de autores clásicos ${ }^{45}$; en el comentario al verso 699, al explicar ut ne, sugiere que se sustituya por $n o n^{46}$; morfológica es también la razón que le mueve a preferir percussit en vez de perculsit en la nota al verso 125 , sobre la que volveremos.

Otras variantes afectan a un cambio en la forma verbal, como observamos en la nota al verso 912, en que, en vez del lactans transmitido, prefiere lactas, que considera más adecuado al contexto ${ }^{47}$, y la del 93 en que considera que conflictatur no es posible por la métrica y propone en su lugar conflictat ${ }^{48}$. En Andria 25 la lectura de Fonseca supone un cambio de persona, pero no solo eso: propone leer pernoscat is en lugar de pernoscatis, y al mismo tiempo sustituir et quid por equid, de todo ello da una breve explicación y alude a lo dicho por un vir doctus ${ }^{49}$.

En ocasiones la variante propuesta supone la supresión de un vocablo, como en el verso 864, en que, basándose en Faernus y el codex Vaticanus, considera más co-

\footnotetext{
41 LANA AC TELA VICTVM QVERITANS. $<v .75>$ : Lanificium magnae laudis fuit foeminis et illo se exerceri pudicitiae erat et probitatis signum, supra in Heautontim. act. 2. sc. $3<282-285>$. "ea res dedit tum existimandi copiam/ nam quotidianae uitae consuetudines/cuiusque ingenium ut sit, declarant maxime/ texentem telam studiose, ipsam offendimus». sic hos uersus restitui cum antea corrupte legerentur. alia de hac re sexcenta suppetunt exempla.

42 Ofrecemos al final un cuadro resumen en que aparecen todas sus ‘intervenciones' en el texto, así como la lectura de las ediciones modernas.

${ }^{43}$ QVID FACIAS ILLI <v. 143>: corrige illo loco illi sic Cicero <epist.> lib. 9. epis. 7. ad Varronem Nam ut audiui de I. Caesare F. mecum ipse, quid hic mihi faciet patri?] itaque legendum [quid facias illo].

${ }^{44}$ La forma está atestiguada en Apuleyo 1, 14 (illo redi).

45 «POTIN》 ES MIHI VERVM DICERE <V. 437>: Alius «pote ne est», recte forte. «potis», et, «pote», quolibet ge-

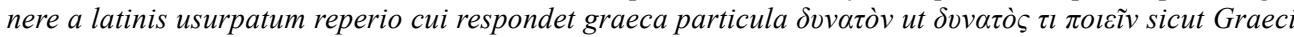
grammatici docent. Y aporta citas de Catulo, Ennio, Lucrecio y Plauto con potis y pote en los tres géneros.


das a me allegatum hunc senem] ubi Donatus [ut ne pro non] ex quo loco facile hunc corriges [《ut ne pater» (ait) «ut ne» pro «ut non»] lege pro «ne non».

${ }^{47}$ EORVM ANIMOS LACTANS <v. 912>: ego potius ex Donato legerem «lactas» sic supra dixerat «inpune facias» $<910>$ et «inlicis» $<911>$.

${ }^{48}$ NAM QVI CVM IN GENIIS CONFLICTATVR <v. 93> Doctissimus Sanchez Brocensis «conflictat» legit, quo uerbo Terentius $<$ Phorm. 505> utitur: et ut uersus constet.

49 VT PERNOSCATIS ET QVID <v. 25>: lege. «ut pernoscat is equid»; sensus erit ut pernoscat is nimirum poeta equid spei sit reliquum. nequaquam ut placuit uiro docto prologum agit poeta sed histrio.
} 
rrecto suprimir el «tu» que hay tras tamen ${ }^{50}$; en 289 considera que es más adecuado et genium tuum, que ofrece Faernus, que la lectura ingenium del texto de Terencio ${ }^{51}$.

Dos veces su discusión se centra en la grafía de un nombre propio: en la Didascalia de la obra propone leer Megalesibus en vez de Megalensibus y lo argumenta con la aportación de varios textos ${ }^{52}$, y sobre el preaenomen de uno de los cónsules que aparecen en la Didascalia, juzga que es Caius, según consta en los Fastos Consulares, en vez del Gneus que le ha llegado ${ }^{53}$.

Pero la mayor parte de las lecturas de que da cuenta suponen un cambio de significado del texto; y también estos son de diferente intensidad: se nos ofrece un cambio de significado muy leve con las variantes: minueris- metueris en el verso 392393, claudier o caluier en el verso $573^{54}$, o deluderes por produceres en el $648^{55}$; más cambio supone symbolam en vez de symbolum (verso $88^{56}$ ) u omina en lugar de

\footnotetext{
${ }^{50}$ TAMEN ETSI HOC VERVM EST. SI. TAMEN. <v. 864>: Omnes ueteres libros scriptos inquit Faernus, et in his antiquiores ita habere [Tamen etsi hoc uerum est? SI. tamen tu] et in Vaticano deesse uocem "tu» quod rectum est, egregius locus si sic legis. [DA. tamen et si hoc uerum est?] Respondet Simo «Tamen». tunc conuertens se ad Dromonem. inquit <865> [Cura ad seruandum uinctum] similis locus est in Carmine obscaeno. $<$ Priapea, 24.34>: Fur abeas, poenam licet indignere, feramque/ Propter olus dicas hoc ego? propter olus.

${ }^{51}$ ET INGENIVM TVVM <v. 289>: in aliis «genium» sic lego nec uiro docto in hac haeremus parte dicit enim «ingenium» naturam mansuetam et bene moratam, Faernus per tuam stabilem naturam sed magno pignore contendam «genium» legi debere, nam quid antiquis augustius Genio. La nota continúa con la explicación de quién es Genio, aportando citas de diversos autores.

52 ACTA LVDIS MEGALENSIBVS. Sic in omnibus fere aeditionibus scriptum inuenio attamen Doctissimus Sanctius Brocensis manu propria, in codice H. S. F. «Megalesibus» notauit, forte respexit ad Liuium et Varronem, qui «Megalesia» scripsit, uerba ipsius uidebis. Cum Romani, enim, Idaeam deorum matrem ex Pessinunte aduocassent, ludos scaenicos quos Megalenses appellarunt instituerunt. Varro «Megalesia» dicta a Graecis quod ex libris Sybillinis accersita ab Attalo, Pergami prope murum Megalesion, ubi templum eius deae, unde aduecta, Romam Festus [«Megalesia» ludos Matris Magnae appellabant] Liuius lib. 36. <36.3> [Per idem fere tempus aedes matris magnae Idaeae dedicata est] et Iterum, $<$ LIV. 36.36.4... $>$ nihil de tempore iudico Nam Appianus < Hann. 233.1ss. > Vidisti quo tempore ludi et qua ratione, non alia e Triuio adducere institutum.

${ }^{53}$ M. MARCELLO G. N. SVLPITIO COSS. In omnibus quos uidi Terentii codicibus sic legitur deprauate; nam collega M. Claudii Marcelli. Caius Sulpitius non GNEIVS sic itaque emendamus ex fastis consularibus Plinio, Cassiodoro. Justino. et Floro. Anno Vrbis DLXXXVIII.

${ }^{54}$ NOLO TIBI VLLVM COMMODVM IN ME CLAVDIER <v. 573>: aliqui legunt. «caluier», et mera somnia narrant ego uero receptam lectionem retineo et pulcherrimo Ciceronis loco confirmo, lib. de offi. $2<2.15>[$ nec ita claudenda est res familiaris, ut eam benignitas aperire non possit, nec ita reseranda, ut omnibus pateat]. Nota que encontramos igual en los folios arriba mencionados del manuscrito 5781.

55 LACTASSES AMANTEM ET FALSA SPE PRODVCERES <v. 648>: Citatur a Lactantio Grammatico in $12<12$, 246>. Thebai. in hunc modum, nisi me lactasses amantem et falsa spe deluderes; exponit blandimentis induceres: forte si sic legisses Terentii scripturam, illinc euocares nam Donatus nec uerbum de producere facit et non abs re si dixeris ex illius commentario uitiatam, ait enim, [Lactasses produxisses oblectasses,] ubi absque dubio tò produxisses in produceres irrepsit cum antea deluderes legeretur uide Nonium in uoce [producere et procudere] et M. Tull. lib. 3. de Oratore. nulla huius uocis in isto sensu mentio. LACTASSES. Accius Medea, $<$ Trag. 414...> ubi Nonius <1.16M...> Pacuui. Iliona $<211 \ldots>$, Caecilius $<$ Statius $>$ Hypobolimeo Rastraria $<91 . .>>$ Acc. Alcmeone $<66 \ldots>$ Varro övo $\lambda$ í $\rho \alpha \varsigma<$ Men. $350 \ldots>$ Cicer. Tuscu. $4<16 \ldots>$.

56 Sobre el comentario a este verso, $c f$. Del Amo-Fortuny (2006). Dice así la anotación del humanista: EHO QVID PAMPHILVS? QVID SYMBOLAM/ DEDIT. <Vv. 88-89>: sensus erit Phaedrum aut Cliniam, aut Niceratum Chrysidem habuisse: Pamphilum symbolam dedisse et cenasse, sed nihil cum Chryside habuisse, (ut ipsius Terentii uerbis utar) nunc uideamus quid sit symbola ubi scio doctissimos uiros halucinasse. sciendum igitur, coe-

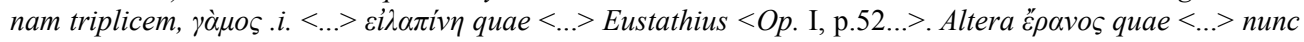


omnia en el verso 96-97; y también cambia el significado del texto con la lectio que dice ser admitida por algunos en el verso 495, en que leen una disyuntiva aut, en lugar de la negación, haud $^{57}$; una nota en la que se extiende nuestro humanista explicando cómo sería el sentido, da cuenta de la aposiopesis que está presente en el texto $\mathrm{y}$ defiende que, según lo que a su juicio hay que sobrentender, la lectura correcta sería con el adverbio.

No omite tratar quién debe pronunciar determinada frase, como en Andria 906, en que, poner saluos sis en boca de Cremes, y no en la de Critón, le obliga a entender que el vocativo que hay a continuación es Crito y no Chreme; añade que hay quienes en vez de certe is est leen eccere is est, un juramento-dice-con el que se invocaría a Ceres ${ }^{58}$.

La nota más larga referida a Andria está dedicada al verso 885 de la obra: eodem die istuc verbum in te accidit, que, transmitido por Prisciano como nuncne demum istud verbum in te incidit, fue corregido por Marcilio así: nuncne demum istud ver in te incidit ${ }^{59}$.

Pasamos a analizar tres notas, tres lugares para los que Fonseca nos ofrece más de una forma, y que hoy mantienen una lectura unánime. Son Andria 96 (omnia y omina), 125 (percussit y perculsit) y 392 (minueris y metueris).

Empezamos por el comentario a los versos 96-97: tum uno ore omnes omnia bona / dicere. Omnia es la lectura que aparece en todas las ediciones actuales de Terencio, pero en el humanismo se propuso leer omina. Así es la anotación de Fonseca:

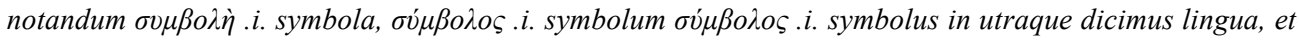


in foemina aliud in neutra declinatione, erit itaque symbola ut ait Proculus $<$ ad Hes. Op. 720 y $722 . .>>$ uidis-

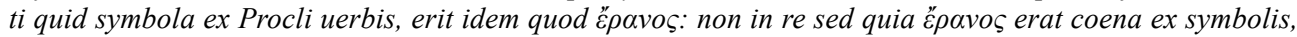
hoc est, portionibus constructa, ut ex Homeri interprete demonstrauimus, hoc est $\delta \varepsilon i \pi v v o v \dot{\alpha} \pi \dot{o} \sigma \nu \mu \beta 0 \lambda \tilde{\omega} v$.


$541 \ldots>$. Plinius. lib. $31<33.4 \ldots>$. et iterum $<$ PLI. nat. 33.6> Plautus Bacchidibus $<327-329 \ldots>$ fuit itaque symbolum quidquid arrae loco in pactionibus dabant unde prouerbium... itaque in Andriae uersiculum symbolam leges, ut obseruarunt doctissimi uiri, et conuiuium erit ex ipsis conflatum. $<\ldots>$ Doctissimus Sanctius Brocensis ut suo uidi in codice, symbolum (contra Turnebum) legendum existimauit sed hodie nihil illi credemus alioqui doctus.

${ }^{57}$ SE IPSVS FALLIT HAVT EGO. $<v .495>$ : aposiopesis. deest «illum decipio» nam ut docto placuit uiro legere nimirum. «aut ego» ut dicat «aut ego fallor» uel «haut ego fallor» error erit. supradixerat Simo <493>: Quem tam aperte fallere incipias dolis. ideo «haut ego ipsum», suple, fallo.

58 CERTE IS EST SALVOS SIS CHREME. $<\mathrm{v}$. 906>: male est deprauata Parisiensis aeditio Hadriani Perier anno MDCI dele Critonis ${ }^{59}$ nomen ante, "saluos sis», colocatum et "Crito» non "Chreme» legendum ita ex alio libro $<906-907 . . .>$ Alii ubi certe is est, «eccere is est» reponunt, Iusiurandum Romanis notum et in Plauto milies dictum, Festus <Paulus Diaconus p.68.16-17> [eccere Iusiurandum est, ac si dicatur per Cererem, ut aecastor aedepol, alii eccere pro ecce positum accipiunt].

${ }^{59}$ EODEM DIE ISTVC VERBVM IN TE ACCIDIT. <v. 885>: Citatur a Prisciano lib. 1. c. 16. <16.102...> Quam lectionem obseruauit olim Marcilius puram et putam Terentii esse. sed haud inducor credere, proprior illi uulga-

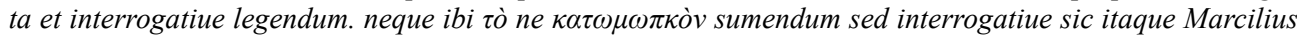
hos corrigit et distinguit. $<$ Andr. 882-885>: $<882>$ PA. me miserum, SI. modone id demum sensi Pamphile/ $<885>$ nuncne demum istud uerbum, uere in te incidit? / $<883>$ olim istuc, olim, cum ita animum induxti tuum/ <884> Quod cuperes aliquo pacto efficiundum tibi. interrogatio autem illa nuncne demum etc. eiusmodi Hactenus de Marcilii emendatione. 
TVM VNO ORE OMNES OMNIA/ BONA DICERE < VV. 96-97>

pro uno consensu, passim apud scriptores Seneca. epist. $50<81,31>$. [in tanta (inquit) iudiciorum diuersitate, referendum bene merentibus gratiam, omnes, uno tibi quod aiunt ore, affirmabunt: Aristopha. equitibus $<670>$.

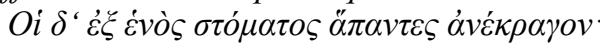

ore uniuersi clamitabant uno simul.

monueram de Terentii emendatione hoc in loco meum Ioannem Baptist. Salazarium Gaditanum, cum D. Laurentius a Prado. Ioannis Scoppae collectaneas commodauit ubi ita correctum inuenio. Lego omina non [omnia] nullum inde eliciens sensum. et



Omnia está unido a bona, que complementa a dicere con el sentido (así lo recogen los traductores) de «dar parabienes», «decir todo bueno»; pero a Fonseca, como antes le ocurriera a Scoppa (1508), no le parece el sentido más adecuado. Nuestro humanista -asegura él mismo- había hablado con su amigo Juan Bautista Suárez de Salazar sobre la corrección de este lugar ${ }^{60}$ de Terencio, cuando Lorenzo Ramírez de Prado le prestó los Collectanea de Scoppa, y allí encontró el término corregido en omina, lo cual considera Fonseca correcto; y comenta al respecto, como lo había hecho Scoppa (y usando la misma juntura elicere sensum), que con omnia no le encuentra sentido; con la lectura omina, continúa, se entendería que pronuncian palabras de buen augurio, o sea, que desean felicidad (laeta precari) a Simón, el padre del joven protagonista; no obstante, finaliza la nota a este lugar con un «pero todavía no me agrada mucho» ${ }^{61}$.

Esto es lo que Fonseca leería que había comentado Scoppa, quien habría dicho en 1517 que con la lectura omnia no era capaz de encontrar un sentido que le «pareciera conveniente»; por eso añade que consideró finalmente que había que leer omina (tandem omnia legendum duxi), y finaliza su argumento manifestando que incluso apostaría que es este el sentido (hunc sensum vel magno pignore contenderem); a continuación va Scoppa parafraseando los términos de los versos en que omnia-omina está inmerso: considera uno ore semejante a consensu, aclara que dicere está por dicebant, y entiende que omina bona sería equivalente a mea auguria, así como fortunas querría decir divitias meas. Concluye con un enérgico dixit bona omina y lo apoya en que omen es un término neutro susceptible de interpretaciones positivas y negativas ${ }^{62}$.

En los editores y traductores modernos la conjetura no ha tenido ninguna trascendencia, si bien en las traducciones ${ }^{63}$, incluso con la unánime lectura omnia, se refleja lo que con omina quisieron ver primero Scoppa y luego Fonseca.

\footnotetext{
${ }^{60}$ Ya hemos mencionado que eran las lectiones asunto frecuente en su correspondencia con humanistas y que en concreto con este había tratado sobre un lugar de Catulo 66.

${ }^{61} \mathrm{Y}$ añadido por otra mano podemos leer: nec mihi.

62 quoniam omen medium est vocabulum, et in bonum, et in malum sonat, ut malum evitaret.

63 «Yo me alegraba de esto; además, todos con voz unánime, me daban parabienes y alababan mi suerte de tener un hijo dotado de semejantes disposiciones» (L. Rubio, 1957). «De ello me alegraba, pero además todos al unísono me daban todo tipo de parabienes y alababan mi suerte por tener un hijo dotado de tal carácter» (J. Román Bravo, 2001). «Esto no sólo me complacía a mí, sino que todos a una me colmaban de parabienes celebrando mi suerte por tener un hijo adornado de tales cualidades» (G. Fontana Elboj, 2008).
} 
En el comentario al verso 125, a propósito de percussit, también habla Fonseca de más de una lectura. Hay varias posibilidades, aunque con poca diferencia entre ellas en lo que se refiere al sentido; pone nuestro humanista como lema el principio del verso: perculsit ilico animum. Existen cuatro lecturas para este lugar: perculi, la única vez en la que el verbo está en primera persona, lectio que presenta algún manuscrito, pero que no aparece reflejada en el humanismo; perculit, sobre la que consideraba Pedro de Figueroa ${ }^{64}$ que era lectura de Despauterius ${ }^{65}$, y las dos más comentadas entre los humanistas: percussit y perculsit. Se trata de perfectos, bien de percutio (percussit) o de percello (las demás), verbos, como se sabe, de significado muy cercano.

Hoy todas las ediciones muestran el perfecto de percutio, mientras que han descartado perculsit probablemente por ser un perfecto poco frecuente de percello; formado a partir del tema del supino, está «atestiguado» solo dos veces en Amiano Marcelino ${ }^{66}$. Mas no fue así de exiguo el uso de este perfecto durante el humanismo, ya que -como veremos- aparecía también en textos de Lucano, Horacio y Tácito. Si nos centramos en Andria, la lectura perculsit fue muy frecuente en las ediciones antiguas: así estaba en Stephanus, Doletus o Pedro de Figueroa, frente al percussit que habían elegido otros editores, entre los cuales se hallan, siguiendo a Donatus, Fabricius o Erasmus.

Y esta es la nota que Fonseca ofrece sobre la lectio: percussit lege, perculsit non est Latinum ut in ora libri habet Sanctii codex.

Es decir, a juicio de nuestro humanista, hay que leer percussit, como está de mano del Brocense en el margen de la edición de Terencio que manejaba, porque, como decía, perculsit no es latino.

Vemos, por tanto, que en esta ocasión no es como otras veces el sensus lo que le lleva a elegir una lectura, sino la «latinidad», argumento que era necesario poner de manifiesto en el caso de la forma percussit, ya que no era evidente para todos. De manera similar criticó Lambinus la lectura perculsit de Horacio, Carmina I, 7, 11, donde hoy se lee percussit ${ }^{67}$. Así mismo se rechazó el perculsit (hoy percussit) que se hallaba en algunas ediciones de Lucano, Farsalia 2, 234, como la de Stephanus de $1545^{68}$ o la de Gryphius de $1569^{69}$. Y tampoco la lectura perculsit en Tácito (hist. 4, 66) ha sobrevivido, sino que ha sido sustituida por el participio perculsis $^{70}$.

\footnotetext{
${ }^{64}$ Pedro de Figueroa (1569, pp.79-80).

${ }^{65}$ No parece que sea así; este gramático de Nínive no afirma -creemos- que prefiere otra lectura para este lugar de Andria (de hecho no encontramos que él hable de perculit como lectura sustitutoria de perculsit), sino que prefiere que el perfecto de percello sea perculi (y no perculsi); cf. Despauterius (1536, p.153).

${ }_{66}^{66}$ Амм. 17, 8, 4 y 25, 8, 13.

${ }^{67}$ «percussit: sic legendum, non perculsit, ut habent vulgati, et nonnulli scripti cod. quod verbum ne Latinum quidem est. nam a verbo percello, praeteritum unum est, perculi». Horacio: Lambinus (1557, p.25).

${ }^{68}$ Lucano: Stephanus (1545, p.33).

${ }^{69}$ Lucano: Gryphius (1569, p.41).

${ }^{70}$ Efectivamente, así está en las ediciones modernas; la variante, respecto a perculsit, atañe a otros elementos del texto. Con perculsit se lee: ingens rerum tumultus perculsit civitatem (o ... cumulus perculsit civitates), mientras que con perculsis hallamos: ingens rerum, perculsis civitatum animis.
} 
Sin embargo, el rechazo de esta forma de perfecto de percello no era unánime en la época. Nuestro Pedro de Figueroa ${ }^{71}$, a pesar de que Ioannis Despauterius ${ }^{72}$ prefería que el perfecto de percello fuera perculi, manifiesta que se alinea con Nebrija y confiesa que opta por perculsit ${ }^{73}$.

También otro humanista, Menenius ${ }^{74}$, aprovecha para decir en su comentario que el verdadero perfecto de percello es perculi, a pesar de lo que se ha transmitido en este lugar terenciano o en otro de Tácito (el ya mencionado hist. 4, 66).

Con esta breve nota Fonseca participa de una discusión extendida en su tiempo acerca de cuál es el perfecto de percello ${ }^{75}$ que se adecua mejor a la lengua latina; además muestra consideración hacia la sugerencia del Brocense ${ }^{76}$, ya que la lectura que defiende la ha encontrado en un codex del maestro extremeño. A su interés por las cuestiones sobre el texto se une en este pequeño comentario la preocupación por la «latinidad» de las palabras.

Por último, nos referimos a la nota a los vv. 392-393: Nec tua causa minueris/ haec.

En este punto de la obra Davo comunica a Pánfilo que el anciano Cremes, tras hacerse pública la relación del joven con Glicera, ya no está dispuesto a concederle la mano de su hija Filomena. Piensa Davo que Pánfilo debe ahora aparentar ante su padre que está dispuesto al matrimonio con esta joven.

A este momento pertenecen las palabras de la comedia que Fonseca pone en el lema: Nec ea causa minueris haec quae facis. Y cuyo significado sería: «no dejes de hacer lo que estás haciendo por este motivo»; el texto continúa: ne is mutet suam sententiam («para que Cremes no cambie de opinión»). Y este es el comentario completo de nuestro humanista: libenter de isto loco male sentio et leui coniectura corri-

\footnotetext{
71 Pedro de Figueroa (1569, pp.79-80).

${ }^{72}$ Tras una cita de Virgilio (Aen. 5.446) que tiene perculit, aporta la de Tácito (HIST. 4.66) con perculsit; y continúa: prateritum fecit perculsi, ut Vello vulsi. sic Terentius (ut notauit Nebrissensis) in Andr. perculsit illico animum. Y concluye: «Libentius dicam perculi»; $c$. Despauterius (1537, p.153).

${ }^{73}$ «Antonius Nebrissensis vir acutissimo ingenio suis in commentariis in verbo Percello, citat hunc Terentii locum perculsit, etsi Ioannis Dispauterii perculit, non autem perculsit, sit sententia, sed cum nostro Nebrissensi libentius dicam perculsit», loc. cit. Declaración que el humanista valenciano realiza después de señalar que hay ediciones que tienen percussit, como leyó Donato, y de aportar un texto de Lucano en que se lee este pretérito perculsit que él elige.

${ }^{74}$ Menenius (1552, p.118).

${ }^{75}$ La polémica continuó mucho tiempo después: en una gramática del siglo XIX, al hablar del perfecto perculsi, aún se cita este lugar de Andria, además del de Horacio, detrás del de Amiano Marcelino, si bien se finaliza con un «but the true reading in the two last passages is percussit»; cf. Cleveland (1845, p.140).

${ }^{76}$ Sánchez de las Brozas es mencionado varias veces en las Notae, y casi siempre a propósito de alguna lectura. Ya en el comentario del primer lema (Acta ludis Megalensibus) nos informa Fonseca de que está viendo una edición de Stephanus con anotaciones del Brocense: Doctissimus Sanctius Brocensis manu propria, in codice H. S. F. Megalesibus notauit; también alude a sus correcciones en otras notae: v. 88: Doctissimus Sanctius Brocensis ut suo uidi in codice, symbolum (contra Turnebum) legendum existimauit; v. 93: Doctissimus Sanchez Brocensis conflictat legit; en el v. 205, sobre una cita de Epídico (664-665) de Plauto, afirma: Sanctius Brocensis in his omnibus legendum putat «hoc» non «haud» ut et in Hecyra <306>. Por lo demás, la única ocasión en que lo menciona para otra clase de comentario es en la nota al verso 782, acerca de la antiphrasis que en él advierte.
} 
gerem, metueris pro minueris ut sit sensus, nec tu ea causa metueris, ob id intellige quod facis. etc. melius forte.

Fonseca refiere que no le acaba de gustar lo que se explica sobre este pasaje y que se atreve a corregirlo con una leve conjetura, metueris en lugar de minueris; de este modo el sentido es: "y tú, por este motivo no temas», sobrentendiendo: "por esto que estás haciendo», etc. Y concluye la nota con un tímido: «quizá mejor» (melius forte).

No es su afán que su conjetura se imponga, sino que la sugiere como una posibilidad que quizá aclare el sentido.

En el humanismo solo se conocía la lectura minueris, y es la que hoy tienen todas las ediciones modernas consultadas; si bien sabemos que había manuscritos con metueris, pero da la impresión de que esta lectura no era conocida en la época de Fonseca, según muestran los ejemplares que hemos visto de los siglos XVI y XVII. Curiosamente Petrus Menenius en 1552, aun poniendo y comentando minueris, informa de que Petrus Nanus ${ }^{77}$ había propuesto leer timueris, y añade la explicación de este humanista belga, quien sobrentiende un $o b$ tras timueris.

Dos humanistas han coincidido en que es mejor un verbo de temor, pero no así los editores modernos, ya que todos se inclinan por minueris, aunque, como consta en los aparatos críticos de sus ediciones, las dos lecturas, minueris y metueris, estaban, como hemos recordado, en algunos manuscritos.

Así pues, como buen humanista, Fonseca no ha eludido tratar cuestiones textua1 les ${ }^{78}$, pero estas no ocupan en frecuencia un lugar destacado. Son poco más de treinta notas de las doscientas quince aproximadamente que constituye su comentario.

Casi siempre manifiesta cuál es su lectura del texto, pero en ocasiones se limita a transmitir que hay quienes lo leen de otra manera, sin tomar partido, solo nos lo presenta y manifiesta un tímido malim, neuter recte, nondum belle mihi arridet.

Y entre las ocasiones -la mayoría, repetimos- en que muestra qué lectura prefiere, tampoco actúa siempre con la misma actitud hacia quienes ven el texto de otra forma; o, podríamos decir, no siempre, su opción es lo mismo de radical. A veces solo un deprauata (906) o deprauate (en la didascalia comentando Gneius, o en la

\footnotetext{
${ }_{77}^{77}$ Se trata del humanista belga que suele también llamarse Nanninck y Nanning. $C f$. Nanius (1548, p.17).

${ }^{78}$ También otros humanistas atendieron a la «fijación» del texto del autor comentado. Entre los que comentan Andria conocemos un trabajo dedicado casi solo a ello: el de Faernus (1587), que evidentemente contiene gran información acerca de las diversas lectiones, o Fabricius ( $c f$. Muretus 1580), si bien nada hay en los lugares en que Fonseca las ofrece. De los demás humanistas que se ocuparon de la comedia terenciana con anterioridad al que presentamos hay muchos que prestan poca atención o ninguna a los comentarios de tipo textual. Tal es el caso de los comentarios de Erasmus (1538), Stephanus (1549), Menenius (1552) o Antesignanus (1560); algunos de ellos aportan en ocasiones alguna variante precedida de un legitur et; en el caso de nuestro Pedro de Figueroa, encontramos alguna variante textual en el margen del texto que acompaña a sus Enarrationes, pero son muy escasas las explicaciones más amplias: una de las más extensas es la ya aludida a propósito de perculsit-percussit (en otros casos la nota es más breve: alii interrogative legunt, p. 55 o haec vera lectio, p. 96, a propósito de neque tu haud del verso 205). Una presencia un poco mayor hallamos en las Observationes de F. Lindenborgius (1573). Entre los comentaristas antiguos, Donatus hacía anotaciones semejantes a las de Fonseca, aunque no en los mismos lugares, mas no así Eugraphius.
} 
nota al verso 904), ego potius (912), rectum (864) son términos que nos hacen ver claramente cuál es su postura, o el que se avenga mejor al texto, a la métrica (93) o a la latinitas. Términos como corrige, lege o legendum también los hemos hallado; en ocasiones solo un melius forte o un «a mí no me acaba de convencer», pero lo más curioso es cómo se refiere a veces a quienes a su juicio han errado en la lectura o interpretación: et mera somnia narrant (v. 573).

Por otra parte, cuando ha criticado una conjetura y ha tomado postura, le han servido como fuente o argumento algunos manuscritos y ediciones (Vaticanos, v. 864), o autores clásicos como Ennio (437), Plauto (88, 437), Catulo (437), Cicerón (143, 573), Varrón (143), Lucrecio (437), Virgilio (885), Festo (289), Séneca $(96,289)$ y Plinio (Gneius, 88, 289); gramáticos antiguos: Donatus (648, 699, 912), Lactancius (648) y Priscianus (885); asimismo ha tomado en consideración a algunos humanistas, entre ellos: Robertus Constantinus (88), Faernus (289), Scoppa (96), Turnebus (96), Marcilius (885); y también los Fastos consulares (Gneius).

A pesar de la poca vigencia de la mayoría de sus propuestas, las notas de tipo textual de Fonseca nos permiten conocer cuál era el Terencio que leían los humanistas, a través de las discusiones filológicas en que ha participado, o bien mediante la información que transmite. Y, sobre todo, somos testigos de las preocupaciones textuales de nuestros humanistas, sea cual fuera el éxito de sus opciones, y en concreto las de D. Juan de Fonseca y Figueroa. El cuadro que aportamos de las variantes textuales comentadas por este humanista sirve de claro ejemplo.

I.- Lugares de Terencio ${ }^{79}$

\begin{tabular}{llll}
\hline Lugar & Ediciones modernas & Lecturas humanismo & Preferencia Fonseca ${ }^{80}$ \\
\hline didascalia Andr. & Megalensibus & Megalensibus-Megalesibus & Megalesibus \\
didascalia Andr. & Gn. Sulpicio & Gn.-C. Sulpicio & Caius Sulpicio \\
Andr. 25 & pernoscatis ecquid & pernoscatis et quid & pernoscat is equid \\
& & pernoscat is equid & \\
Andr. 88 & symbolam & symbolam-symbolum & symbolam \\
Andr. 93 & conflictatur & conflictatur-conflictat & conflictat \\
Andr. 96 & omnia & omnia-omina & omina \\
Andr. 125 & perculsit & perculsit-percussit-perculit & percussit \\
Andr. 143 & illi & illi-illo & illo \\
Andr. 289 & ingenium & ingenium-genium & genium \\
Andr. 392 & minueris & minueris - timueris & metueris
\end{tabular}

\footnotetext{
${ }^{79}$ No incluimos en la tabla la corrección a Heautontimorumenos, 282-285, versos hasta entonces «corruptos», según sus propias palabras.

${ }^{80}$ Recordamos que no siempre Fonseca manifiesta cuál es la lectio que prefiere.
} 


\begin{tabular}{|c|c|c|c|}
\hline Andr. 437 & potin & potin-pote & pote \\
\hline Andr. 495 & haud ego & haut ego - aut ego & aut ego \\
\hline Andr. 573 & claudier & claudier-calvier-interclaudier & claudier \\
\hline Andr. 648 & produceres & produceres - deluderes & \\
\hline Andr. 699 & ut ne & ut ne-utnon & ut non \\
\hline Andr. 864 & tamen tu & tamen tu-tamen & tamen \\
\hline Andr. 885 & $\begin{array}{l}\text { eodem die istuc verbum } \\
\text { vere in te accidit }\end{array}$ & $\begin{array}{l}\text { eodem die istuc verbum } \\
\text { in te accidit (Prisciano) } \\
\text { nuncne demum istud verbum } \\
\text { in te incidit } \\
\text { nuncne d. } i . v \text { in te incidit? }\end{array}$ & \\
\hline Andr. 906 & CR. saluos sis, Chreme & $\begin{array}{l}\text { CR. saluos sis, Chreme } \\
\text { CH. saluos sis, Crito }\end{array}$ & CH. saluos sis, Crito \\
\hline Andr. 912 & lactans & lactans - lactas & lactas \\
\hline
\end{tabular}

\section{II.- Lugares de otros autores latinos}

\begin{tabular}{|c|c|c|c|c|}
\hline Nota Andria & Lugar & Ediciones modernas & Lecturas humanismo & Fonseca \\
\hline didascalia & SymM. epist. 1, 31, 3 & Ambivio & Ambitio - Ambivio & Ambivio \\
\hline 79 & GLOSA & & quaestoria & quaestuaria \\
\hline 205 & $\begin{array}{l}\text { Plavt. Bacch. } 1035 \text {, } \\
\text { Epid. } 664 \text { Hec. } 306\end{array}$ & haud & haud-hoc-at & $h o c-a t$ \\
\hline 211 & SEN. epist. 88,8 & [in]pudica & impudica-pudica & pudica \\
\hline $272-3$ & MART. 2, 2, 3 & percisum & praecisum-percisum & praecisum \\
\hline 442 & GLOSA & & pucatrix & putatrix \\
\hline 457 & Svet. Iul. 47 & rectiora & recentiora - decentiora & recentiora \\
\hline 464 & Mela 1,45 & colant & colant - alant-tollunt & tollunt \\
\hline 505 & LVCIL. sat. 2, 13 & moetino & $\begin{array}{l}\text { mutino - moetino } \\
\text { mutonio }\end{array}$ & $\begin{array}{l}\text { mutino } \\
\text { moetino }\end{array}$ \\
\hline 568 & SEN. dial. 6, 9 & si admoneatur ut & $\begin{array}{l}\text { si a. ut suis - si a. de } \\
\text { his - sive a. et }\end{array}$ & \\
\hline 726 & SERV. Aen. 12,120 & ros marinus & ros maximus - ros marinus & ros marinus \\
\hline 904 & Dıом. 3, 490 & facerent & facerent & faceret \\
\hline 904 & MART. $6,6,1-2$ & quattuor & quatuor & quartum \\
\hline
\end{tabular}


Además en la nota al verso 459 sugiere que un texto de Gelio $(11,6)$ podría ser cambiado en algo, según un manuscrito que él mismo posee; pero no especifica cuál sería la corrección. Y también en la nota al v. 473 habla de una conjetura a un texto de Festo, mas no detalla cuál es el lugar que propone enmendar.

III.- Lugares de autores griegos

\begin{tabular}{|c|c|c|c|}
\hline Nota Andria & Lugar & Lectio transmitida & Fonseca \\
\hline 161 & AESCHINES, Ep. 109, 5 & $\dot{\varepsilon} \pi v \tau \rho \varepsilon ́ \psi \varepsilon ı v$ & $\dot{\varepsilon} \pi \imath \tau \rho \dot{\varepsilon} \psi \varepsilon ı v$ \\
\hline 473 & Pi. N. 7, 1; THEOC. 17,61 & 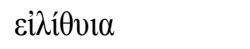 & cì $\varepsilon^{i} \theta v i \alpha$ \\
\hline 483 & MENANDER & 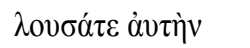 & $\lambda o v \sigma \alpha ́ \tau \varepsilon \dot{\alpha} v \tau \grave{\eta} \nu \tau \alpha^{\prime} \chi 1 \sigma \tau \alpha$ \\
\hline 726 & LiB. Decl. 6, 28 & oikías $\beta \omega \mu$ ós & oikías $\beta \omega \mu$ ó \\
\hline 726 & PoL. $4,125,9$ & $\dot{\alpha} \lambda 1 \varepsilon \dot{\varsigma} \varsigma$ &  \\
\hline
\end{tabular}

\section{REFERENCIAS BIBLIOGRÁFICAS}

\section{HUMANISTAS}

\subsection{TERENCIO}

Antesignanvs (1560), Terentius in quem triplex edita est P. Antesignani Rapistagnensis commentatio, Lugduni, apud Mathiam Bonhome.

FAERnvs (1587), Gabrieli Faerni Emendationes in sex fabulas Terentii.

FigueroA, P. DE (1569), Petri a Figueroa enarrationes vere aureae in P. Terentii Andriam et Eunuchum, Valentiae, ex typographia Petri a Huete.

Menenivs (1552), Petri Menenii Lugdunensis Commentaria in P. Terentii Andriam et Eunuchum. Lugduni, apud Ioan. Tornaesium et Gul. Gazeium.

Mvretvs (1580), M. Antonii Mureti, argumentorum et scholiorum in Terentium liber. Francisci Fabricii Marcodurani, in sex Terentii comoedias Annotationes, Theod. Pulmanni Craneburgii Variae lectiones sive emendationes potius, Eographii fragmentum Commentarii in Terentium, Veterum scriptorum de P. Terentio Afro testimonia, Ab Aldo Manutio, Paulli F. collecta, Aldi Manutii Paulli F. Aldi N. De tibiis, Antuerpiae, ex officina Christophori Plantini.

Stephanvs (1529), P. Terentii Comoediae sex, tum ex Donati commentariis, tum ex optimorum, praesertim veterum, exemplarium collatione, diligentius quam unquam antehac, emendatae. Aelii Donati antiquissimi et celeberrimi grammatici in easdem, quicunque extant, commentarii, ex veteri codice manu descripto, graecis etiam prepositis, accurate castigati. Calphurnii in tertiam comoediam doctissima interpretatio. Eorum, quae in commentariis sparsim annotata sunt, index amplissimus, Parisiis, ex officina Roberti Stephani

\subsection{OTRAS OBRAS}

Correa, G. (1622), Commentatio seu Declaratio ad illud Geneseos "Sed fons ascendebat e terra, irrigans uniuersam faciem terrae”, capite secundo. Vbi etiam illud D. Matthaei, 
vespere autem Sabbati, capite ultimo; et alia obiter explicantur. AVTHORE MAGISTRO Gonzalo Correas, Linguarum Hebraicae et Graecae Salmanticae primario. Salmanticae. Apud Antonium Vazquez.

DesPavterivs (1536), Iohannis Despauterii Ninivitae Commentarii Grammatici, Parisiis, Ex officina Roberti Stephani.

Lambinvs (1557), Dionysii Lambini Monstroliensis. regii professoris in Q. Horatium Flaccum ex fide atque auctoritate complurium librorum manuscriptorum... commentarii copiosissimi, Francofurti ad Moenum, ex officina typographica Andreae Wechelli.

Gryphivs (1569), M. Annaei Lucani, De bello ciuili libri decem, Lugduni, apud Antonium Gryphium.

Stephanvs (1545), M. Anneii Lucani De bello ciuili libri decem, Lutetiae, ex officina Rob. Stephani typographi Regii.

Mvssambertvs, CL. (1607), In L. Ramiresii ad M.V. Martialem hypomnemata, Parisiis, exc. Dionysius Langlois.

Nanivs (1548), Petri Nannii Alcmariani in Collegio Buslidiano apud Louanienses Latini professoris, sive Miscellaneorum decas una. Louanii, ex officina Servaii Sasseni.

RAMírez De PrAdo, L. (1607), Hypomnemata ad lib. Spectaculorum et quatuor primus Epigrammatom M.V. Martialis $<\ldots>$, Parisiis, apud Michaelem Somnium.

Scoppa (1508), Lucii Ioannis Scoppae Parthenopei grammatices institutiones et in calce epitome cum metrorum arte pro pueris quam epitomen separatam quoque imprimendam curauimus, Neapoli.

\section{ESTUDIOS}

AHUS: Archivo Universitario de Sevilla.

AJo y SÁINZ DE ZÚÑIGA, C. Ma (1972), Historia de las Universidades hispánicas, orígenes y desarrollo, vol. 8.

Amo Lozano, M. (2009), «Cuestiones retóricas en el comentario de Fonseca y Figueroa a la Andria», en Arcos Pereira, T.-Fernández LóPez, J.-Moya del Baño, F. (coords.), Pectora mulcet: estudios de retórica y oratoria latinas, Logroño, Instituto de Estudios Riojanos, vol. II, pp. 927-940.

Amo Lozano, M.-Fortuny Previ, F. (2005), «Terencio explica a Terencio. Las citas terencianas en el comentario de Juan de Fonseca a Andria», Myrtia 20, 223-241.

Amo Lozano, M.-Fortuny Previ, F. (2006), «Terencio, Andria 88: El comentario de Juan de Fonseca», en Calderón, E.-Morales, A.-Valverde, M. (eds.), KOINÒS LÓGOS. Homenaje al profesor José García López, Murcia, Universidad, vol. I, pp. 5-61.

Amo Lozano, M.-Moya del Baño, F. (2008); «Dos epístolas «filológicas» con Andria de tema. De Fonseca y Figueroa a Solórzano Pereira y respuesta de Solórzano a Fonseca», Myrtia 23, 287-314.

AUSA: Libros de actas de la Universidad de Salamanca.

Beltrán Noguer, M ${ }^{\mathrm{a}}$ T. (2007-2008), «Sobre una edición inédita de la Andria terenciana», Estudios Románicos, Homenaje al Profesor Joaquín Hernández Serna, Murcia, Universidad, vol. I, pp. 261-266.

Castro de Castro, J. D. (2007), «El comentario a la Andria (1569) de Pedro de Figueroa: sus fuentes», Myrtia 22, 181-205.

Charlo Brea, L. (2007), «Nota crítica de Suárez de Salazar a Catulo 66, 52-58», Myrtia 22, 207-222. 
Cleveland, C. D. (ed.) (184533), Grammar of the latin language of A. Adam, Philadelphia. GiL FERNÁNDEZ, L. (1984), «Terencio en España: del Medievo a la Ilustración», en Estudios de humanismo y tradición clásica, Madrid, pp. 95-125.

La Barrera y Leirado, C. A. de (1867), Poesías de don Francisco de Rioja, Madrid.

López Bueno, B. (1984), Francisco de Rioja, Poemas, edición de, Madrid.

LóPEZ NAvío, J. (1964), «Don Juan de Fonseca, canónigo maestrescuela de Sevilla», Archivo Hispalense 41, 83-126.

Miralles Maldonado, J. C. (2010), «Fonseca, poeta latino», en IV Congreso Internacional de Humanismo y pervivencia del mundo clásico, Homenaje a A. Prieto, I, pp. 1641-1654.

Moya del BAÑo, F. (1986), «Los comentarios de J. de Fonseca a Garcilaso», en Actas de la IV Academia Literaria Renacentista, Garcilaso, Salamanca, pp. 201-234.

MoYa DEL BAÑo, F. (1988), «Don Juan de Fonseca y Figueroa y la biografía de Pedro de Valencia del Manuscrito Biblioteca Nacional 5781», Myrtia 3, 9-17.

Moya del Baño, F. (2006), «La obra de Plinio el Viejo en el canónigo sevillano don Juan de Fonseca», Calamus renascens 7, 147-160.

Moya del Baño, F.-Beltrán Noguer, Ma T. (1987-89), «Las notas de D. Juan de Fonseca a la Jerusalén de Lope», Estudios Románicos 5, 65-84.

Moya del Baño, F.-Fortuny Previ, F. (1990), «Un comentario perdido a Petronio», Myrtia $5,13-22$.

N. Antonio (1783), Bibliotheca hispana nova, Madrid (=Roma 1672), I.

Ortega Castejón, J. F. (1989), «Juan Bautista Suárez de Salazar y su enmienda al v. 54 del Carmen 66 de Catulo. La conjetura de Aquiles Estazo», Actas del VII Congreso Español de Estudios Clásicos, III, Madrid, pp. 629-634. 


\section{APÉNDICE}

\section{OTRAS EDICIONES Y OBRAS HUMANISTAS}

Calpvrnivs Brixiensis, Io. (1538), P. Terentii Comoedias, una cum scholiis ex Donati, Asperi, et Cornuti commentariis decerptis.... Basileae in officina Frobeniana.

Ascensivs (1512), P. Terentii aphri comicorum elegantissimi Comedie a Guidone Juuenale perquam litterato familiariter explanate, et a Jodoco Badio Ascensio una cum explanationibus rursum annotate atque recognite. cumque eiusdem Ascensii praenotamentis atque annotamentis suis locis adhibitis, Lugduni.

Doletvs (1540), Obseruationes in Terentii comoedias nempe Andriam: et item Eunuchum. Steph,. Doleto Gallo Aurelio Autore, Lugduni, Apud eundem Doletum.

Goveanvs (1570), P. Terentii Aphri Comoediae sex: accuratè sanè, \& diligenter emendatae, Annotationibusque Ant. Goveani illustratae. Post omnium editiones summa uigilantia recognita. Venetiis, apud Hieronymum Scotum.

Linderbrvchivs (1726), P. Terentii Afri Comoediae sex... accedunt interpretes uetustiores, Aelius Donatus, Eugraphius, Calphurnius ... porro Frid. Lindenbruchii Obseruationes... curauit Arn. Henr. Westerhouius, t. primus, apud Petrum Gosse.

Mvretvs (1551), Marci Antonii Mureti in Andriam annotationes, Lutetiae, apud Vascosanum. 